\title{
Reflets
}

Revue ontaroise d'intervention sociale et communautaire

\section{Étiologie sociale et santé mentale : ouvertures et fermetures des modèles explicatifs dominants}

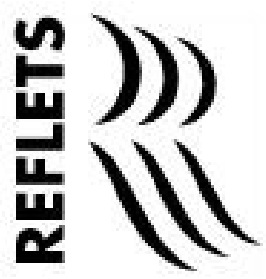

\section{Louise Blais}

Volume 1, numéro 2, automne 1995

La santé communautaire en Ontario français : défis et espoirs

URI : https://id.erudit.org/iderudit/026080ar

DOI : https://doi.org/10.7202/026080ar

Aller au sommaire du numéro

Éditeur(s)

Reflets : Revue ontaroise d'intervention sociale et communautaire

ISSN

1203-4576 (imprimé)

1712-8498 (numérique)

Découvrir la revue

Citer cet article

Blais, L. (1995). Étiologie sociale et santé mentale : ouvertures et fermetures des modèles explicatifs dominants. Reflets, 1(2), 138-162.

https://doi.org/10.7202/026080ar

\section{Résumé de l'article}

La pauvreté est un thème récurrent de l'épidémiologie psychosociale et psychiatrique depuis deux siècles. Cet article examine les divers modèles explicatifs concernant le lien entre la pauvreté et la problématique générale de ce qui est communément appelé la «santé mentale». Il s'agit, en particulier, de dégager les ouvertures et fermetures de ces modèles. Si les approches épidémiologiques peuvent jouer un rôle important en identifiant des indicateurs socio-économiques qui menacent la santé mentale, elles peuvent, par effet pervers, stigmatiser des personnes vulnérables en les enfermant dans une logique de groupes à risque sur lesquels seradéployé le dispositif d'intervention formel. En conclusion, l'article fait appel à la nécessitéd'un recadrage dans l'étude du lien entre santé mentale et facteurs sociaux de manière à faireplace à l'expérience de la pauvreté et à la façon dont les problèmes qui en découlent sontreprésentés, vécus et exprimés dans des contextes spécifiques et différents.
Tous droits réservés (C Reflets : Revue ontaroise d'intervention sociale et communautaire, 1995
Ce document est protégé par la loi sur le droit d'auteur. L'utilisation des services d’Érudit (y compris la reproduction) est assujettie à sa politique d'utilisation que vous pouvez consulter en ligne.

https://apropos.erudit.org/fr/usagers/politique-dutilisation/ 


\section{Étiologie sociale et santé mentale:}

\section{ouvertures et fermetures des modèles explicatifs dominants}

L a pauvreté est un thème réaurrent de l'épidémiologie psychosociale et psychiatrique depuis deux sièdes. $C$ et artide examine les divers modèles explicatifs concernant le lien entre la pauvreté et la problématique générale de ce qui est communément appelé la «santé mentale». II s'agit, en particulier, de dégager les ouvertures et fermetures de ces modèles. Si les approches épidémiologiques peuvent jouer un rôle important en identifiant des indicateurs socio-économiques qui menacent la santé mentale, elles peuvent, par effet pervers, stigmatiser des personnes vulnérables en les enfermant dans une logique de groupes à risque sur lesquels sera déployé le dispositif d'intervention formel. $\mathrm{E} n$ condusion, l'artide fait appel à la nécessité d'un recadrage dans l'étude du lien entre santé mentale et facteurs sociaux de manière à faire place à l'expérience de la pauvreté et à la façon dont les problèmes qui en découlent sont représentés, vécus et exprimés dans des contextes spéafiques et différents.

\section{L ouise B lais}

P rofesseure, É cole de service social, U niversité d'0 ttawa

\section{Introduction}

L'étude du lien entre l'état psychologique d'individus ou groupes et les conditions socio-économiques d'une collectivité a été une préoccupation centrale de l'épidémiologie depuis près de deux siècles. Ce texte examinera les divers modèles élaborés depuis plusieurs décennies pour expliquer ce lien. II s'agira d'une mise en perspective historique de l'épidémiologie psychosociale de manière à voir comment elle s'est construite à travers le temps et 
«L'accent ici portera principalement sur les divers modèles proposés dans la littérature pour expliquer les liens entre pauvreté et état de santé mentale.» l'espace et comment son expansion s'est traduite en un glissement progressif du champ socio-culturel vers celui de la santé publique (que plusieurs nommeront la médicalisation du sodal). $\mathrm{N}$ ous ferons une synthèse de quelques écrits d'un vaste corpus de recherche s'inscrivant dans des courants qui ont dominé, et qui dominent encore largement, la pensée contemporaine quant à l'étiologie sociale du fait mental, selon l'expression de R oger Bastide (1965). L'accent ici portera principalement sur les divers modèles proposés dans la littérature pour expliquer les liens entre pauvreté et état de santé mentale. Le but consiste en un effort d'identification des ouvertures et des fermetures, des enjeux et des limites du modèle déficitaire sous tendant la quasi totalité des études. En conclusion, quelques pistes de réflexions seront amorcées indiquant la nécessité de revoir l'idée que l'on se fait de la santé mentale en milieux de pauvreté et les implications qui en découlent pour la recherche et la pratique.

\section{L'épidémiologie psychosociale en perspective}

L'histoire de l'étiologie sociale de la problématique générale de la santé mentale remonte, en $\mathrm{A}$ mérique du $\mathrm{N}$ ord, à la double révoIution, industrielle et asilaire, qui marque le dix-neuvième siècle. D éjà dans les années 1850, Javis, un médecin du M assachussets, constatait une présence plus importante de patients déments (c'était un des termes utilisés à l'époque, avec folie, aliénation mentale et autres, pour parler de ce qu'aujourd'hui on nomme maladie mentale, troubles mentaux, etc.) issus des couches sociales les plus pauvres sur le plan économique (Fortin, 1987). M ais c'est surtout à partir de la grande crise des années 1930 que l'épidémiologie prend sa véritable envolée. $M$ arsh (1938 dans Fortin, 1987) à M ontréal, Faris et Dunham (1939) à C hicago, Hollingshead et R edlich (1958) à Yale, Leighton (1959), D ohrenwend et D ohrenwend (1969 dans G ibbs, 1980), B renner (1973), B rown et $\mathrm{H}$ arris (1978a) ne sont que quelques-uns des chercheurs qui ont marqué la science épidémiologique du fait 
«L es années 60 ont été un point tournant pour l'étude des phénomènes mentaux dans la population globale » mental depuis quelques décennies. $D$ ans le monde anglo-saxon, on peut dire qu'ils constituent quelques-unes des références à partir desquelles s'est effectuée toute une série de recherches qui se répondent les unes aux autres et dont le but consiste à vérifier les hypothèses existantes concernant les dimensions social es de la santé mentale.

Les années 60 ont été un point tournant pour l'étude des phénomènes mentaux dans la population globale. C eci n'est pas indépendant des nouvelles ouvertures que connaît I'univers de la santé et de la maladie mentale. A près les modernisations de l'appareil psychiatrique qui avaient marqué les années d'après-guerre de la plupart des pays occidentaux, en particulier en Amérique du $\mathrm{N}$ ord, après les remises en question d'anciennes valeurs régissant les comportements et les rôles sociaux, les frontières entre le normal et le pathologique s'avéraient de plus en plus floues. $M$ ais elles ne disparaissaient pas pour autant. $D$ ans la foulée du $R$ apport Lalonde de 1974, et à l'instar de la vision proposée par l'O rganisation mondiale de la santé (I'OMs), on assiste désormais à une définition de la santé à la fois élargie et infiniment plus globale. D'où la notion de la santé comme «un état de bien-être suffisant pour accomplir de façon adéquate un certain niveau d'activités physiques, mentales et sociales, en prenant en considération l'âge» (Lalonde, 1974).

Partant de cette vision profondément fonctionnaliste, le gouvernement fédéral lançait, à la fin des années 70 , une vaste enquête sur la santé des C anadiens (Statistique C anada, 1981).A xée surtout sur la santé physique, cette enquête a néanmoins servi de point de départ à des études épidémiologiques entreprises par la suite par des gouvernements provinciaux. M ais ce qu'il y a de nouveau ici, c'est la place accordée à l'épidémiologie psychoso ciale et à la connaissance détaillée de l'état de santé psychologique de la population. Et il n'est pas un hasard que ces études, du fait qu'elles portent sur de très grands nombres, aient pu être effectuées grâce au nouveau dispositif technologique qui se mettait en place.

$M$ ais il n'y a pas que la taille de ces études qui mérite d'être signalée. Plus fondamentalement, c'est au niveau de la 
«À vrai dire, on a l'impression qu'aucun état d'âme de la vie quotidienne et ordinaire - on n'oserait dire normale - ne peut échapper, désormais, au regard pénétrant et décapant du projet de l'épidémiologie psychosodale» classification nosologique que s'est opéré un changement significatif. Si les catégories classiques de la psychose et de la névrose caractéristiques des anciennes études ne disparai ssent pas entièrement, elles se multiplient de manière exponentielle pour recouper une symptômatologie infiniment plus différenciée. En effet, comme le montre Sarbin (1990:272-273), le premier DSM (Diagnostic and Statistical M anual) publié en 1952 contenait $106 \mathrm{ca}$ tégories diagnostiques; le second, paru en 1968, en contenait 182, le troisième, publié d'abord en 1980 et révisé en 1987 (DSM-III-R ), contenait respectivement 265 et 292 catégories. Le DSM -IV, qui vient de paraitre, en contient autour de 400.

Deux tendances semblent caractériser cette nouvelle épidémiologie. $D$ 'une part, on voit se développer des outils, par exemple le $E$ pidemiologic $C$ atchment A rea (ECA), permettant d'établir des diagnostics psychiatriques spécifiques, voire lourds, dans la population générale. Ainsi, parmi les principaux repérés sont les phobies, l'alcoolisme, les toxicomanies, les dépressions majeures, les personnalités antisociales, la schizophrénie, et d'autres encore (R obins et al.,1991). D'autre part, on voit se développer les échelles pour mesurer une symptômatologie plus légère, tels le bonheur, l'anxiété, l'insatisfaction (dans le mariage, au travail), l'adaptation, l'image de soi, le contrôle de soi, la déviance, le nombre de personnes dans son réseau qui permettrait de mesurer l'intégration sociale, les comportements et idées suicidaires (lointaines et récentes), les événements de vie stressants (mort d'un proche, divorce, chômage, retraite... ), les habitudes de vie (alcool, tabac, sédentarité, alimentation, drogues... ) , les états dépressifs et l'anxiété, le niveau d'énergie, la capacité de concentration, l'ennui, les peurs, l'intérêt sexuel, le contrôle des émotions et autres «indicateurs sensibles» en fonction du sexe, de l'âge, du revenu, de la scolarité, du lieu de résidence, du statut matrimonial, du nombre d'enfants... À vrai dire, on al'impression qu'aucun état d'âme de la vie quotidienne et ordinaire - on n'oserait dire normale ne peut échapper, désormais, au regard pénétrant et décapant du projet de l'épidémiologie psychosociale. Ceci est peu banal si on considère l'importance que prenait pour Foucault la notion de l'aveu dans son histoire de l'enfermement de la folie (Foucault, 
«... à l'instar de Sarbin, on peut dire que le diagnostic de psychopathologie est prinapalement un diagnostic pour les gens pauvres»
1972). N ina Berberova le disait autrement dans L e R oseau révolté (1988) : «Is savent ce qu'ils font, ceux qui organisent la vie des hommes (sic) de manière à leur interdire toute solitude... »

D epuis de nombreuses décennies, à travers des nuances et des différenciations toujours plus subtiles, les études confirment les liens entre la présence de troubles mentaux et un faible niveau socio-économique et culturel. En d'autres termes, et à l'instar de Sarbin, on peut dire que le diagnostic de psychopathologie est principalement un diagnostic pour les gens pauvres (Sarbin, $1990: 266)$. M ais si l'existence de ces liens ne fait aucun doute pour l'épidémiologie psychosociale, des clivages profonds se manifestent dès lors qu'est posée la question de la direction de la causalité. D epuiscent cinquante ans, deux thèses se heurtent pour expliquer les liens: I'une de la sélection naturelle, l'autre de la causalité sociale. En 1850, ayant constaté la plus forte prévalence de démence parmi les pauvres de Boston, Javis attribuait ce lien à leur «infériorité naturelle». À ce darwinisme social s'est opposée la thèse inspirée des philosophies du sodal welfare où l'on attribuait les phénomènes mentaux aux conditions socio-économiques qui les généraient.

\section{Les thèses explicatives}

Les écrits faisant l'objet de la discussion qui suit ont été choisisen fonction de leur valeur explicative par rapport aux dimensions sociales de la problématique santé mentale. Parmi les auteurs auxquels nous nous attarderons, quelques-uns se sont imposés par leur influence certaine, si l'on en juge par la fréquence de leur apparition dans les bibliographies et les banques de données informatisées. À ce titre, on peut dire qu'ils servent de référents autour desquels s'articule un corpus imposant de recherches qui se répondent les unes les autres.

L'opposition entre les thèses de la sélection naturelle et de la causalité sociale en est une, non pas de paradigme, mais de perspective 
de départ. Si au cours des dernières décennies, le raffinement des outils de recherche a permis de rendre plus complexe la chaîne causale en faisant intervenir des variables intermédiaires toujours plus nombreuses et subtiles, à un pôle comme à l'autre, on accepte, du moins implicitement, le modèle médical des désordres mentaux (Brown et Harris, 1978b). D ans ce sens, l'ajout de niveaux de complexité ne peut en rien masquer l'idée première de ce projet qui consiste à faire le départage entre ce qui est, ou n'est pas, un cas.

Cette dominance du paradigme médical dans les études portant sur la problématique de la santé mentale a été mise en évidence dans une analyse récente de deux cents recherches dans le champ de la santé mentale. Ainsi, en examinant les origines disciplinaires des auteurs, Bibeau et son équipe (1989:107) ont pu constater que près des deux-tiers provenaient de disciplines d'orientation clinique et profondément enracinées dans le modèle médical : psychiatres, psychologues, médecins, épidémiologues, etc. II est certain qu'une telle puissance numérique influence les cadres théoriques d'un corpus de connaissance pris dans son ensemble.

\section{Les thèses de la causalité simple}

\section{La sélection naturelle}

À un pôle du débat, il est postulé que la présence plusimportante de psychopathologies au bas de l'échelle socio-économique est due à l'existence d'une vulnérabilité ou de symptômes psychiques déjà présents. Ainsi, les facteurs culturels liés à la classe sociale ne sont pas déterminants dans l'émergence des phénomènes mentaux (B ebbington et al., 1981; H usaini et $\mathrm{N}$ eff, 1981). Si ceux-ci semblent se distribuer de manière inégale dans la population, c'est qu'un jeu de forces naturelles est à l'oeuvre et que les moins aptes seraient plus sujets à l'échec. D'où la dérive sociale. Et la présence plus importante de divers troubles mentaux parmi certains individus et groupes dans la population serait attribuable à des traits de personnalité prédisposants, tels des capacités de débrouillardise ou d'adaptation (coping styles) inadéquates (Liem 
et Liem, 1978), un plus faible contrôle de soi et de son environnement (H usaini et $\mathrm{N}$ eff, 1981), le recours à des stratégies inefficaces, comme par exemple la résignation ou le déni (Pearlin et Schooler, 1978).

Dans une recension des écrits sur l'étiologie sociale de la problématique de la santé mentale, Tousignant (1987) arrive à la conclusion que les thèses de la sélection naturelle résistent aux preuves des thèses de la causalité sociale auxquelles nous reviendrons. Ainsi, selon lui, si les pauvres semblent sur-représentés dans les statistiques sur les troubles mentaux, cela serait dû à un déficit au niveau de caractéristiques personnelles, tels l'estime de soi, l'action, le sentiment de contrôle personnel, l'optimisme (Ibid:107) etc. : «... les personnes dont le statut est... inférieur dans notre société... ne possèdent pas un sentiment de contrôle et de maîtrise de ce qui leur arrive» (I bid:114). Q uant à l'apparente concentration des phénomènes psychopathologiques dans les milieux pauvres, il ne s'agirait pas simplement des facteurs de stress liés aux conditions de travail, aux logements ou aux problèmesfamiliaux. $\mathrm{C}$ ar, comme le remarqueTousignant, elle pourrait tout aussi bien être le reflet de milieux qui constituent des «lieux de refuge pour les personnes à problèmes de toutes les classes sociales à cause d'une banque de logements... à prix modique ou d'une plus forte tolérance à l'égard des problèmes de comportement» (Ibid:125). Enfin, pour ce qui est du lien entre problème de santé mentale, chômage et récession économique, Tousignant est d'avis que les études renseignent très peu sur la part jouée par la perte d'emploi dans l'état psychologique, car «de nombreuses personnes peuvent être au chômage d'abord et avant tout parce qu'elles sont alcooliques, déprimées ou sans motivation» (Ibid:131).

Le problème principal que comportent les thèses de la sélection naturelle nous semble se situer au niveau d'une désocialisation de ces traits de personnalité en les présentant comme des caractéristiques purement individuelles plutôt que comme reflétant l'influence de conditions de contexte et de stratégies de survie appropriées à ces conditions. En outre, il faut se demander quelles valeurs sous-tendent le départage entre sain et malsain. Q uelle 
est l'échelle qui permet d'évaluer une stratégie comme inefficace? $\mathrm{O}$ ù se situe la frontière entre ce qui est efficace ou pas? $\mathrm{Q}$ ue signifie, et comment mesurer, une bonne estime de soi, un bon contrôle de soi? II nous semble que cette négligence à expliciter le cadre de référence à partir duquel on attribue des valeurs différenciées et hiérarchisées à des traits de personnalité eux-mêmes différenciés constitue un problème de fond ayant des conséquences à divers niveaux. Parmi les éléments problématiques qui forment les contours de ces cadres de référence se retrouve le fait, de plus en plus admis (B ronfenbrenner, 1979; Eichler, 1983; Sarbin, 1990; etc.), que les chercheurs, majoritairement de sexe masculin et provenant essentiellement de la classe moyenne, sont eux-mêmes imprégnés de val eursplus ou moinsconsciemment érigées comme références à partir desquelles se construisent les échelles de mesures et les méthodes de recherche pouvant départager entre cas et non-cas. C ette incapacité d'inscrire les comportements, sentiments et attitudes sur le registre des valeurs culturelles - celles des chercheurs incluses - donne lieu à une évaluation purement fonctionnaliste traduisant, en fait, des niveaux de désirabilité ou d'acceptabilité sociales.

\section{La causalité sodiale}

Les thèses de la causalité sociale ont leurs origines dans un autre déterminisme, économique celui-là. Prenant racine dans la pensée marxiste et socialiste du dix-neuvième siècle, le déterminisme économique a très tôt pénétré la science épidémiologique qui accompagnait la lutte du mouvement de la santé publique contre les conditions insalubres du prolétariat. A ppliquées au champ de la santé mentale, ces thèses postulent que la surreprésentation satistique des troubles mentaux au bas de l'échelle sociale trouve sa source dans les dislocations occasionnées par les bouleversements sociaux, entrainnant une perte de cohésion sociale qui affecterait davantage les individus et groupes défavorisés.

L'espace de ce texte nous contraint à un survol rapide de plusieurs décennies de recherches qui s'inscrivent dans le courant de la causalité sociale. C'est surtout dans la période de l'aprèsguerre que l'on voit se multiplier les recherches sur le rapport 
entre l'économie et la psychopathologie. Parmi les premières et les plus importantes de cette période, on trouve celles faites par $\mathrm{H}$ ollingshead et R edlich (1958). En développant des mesures plus directes et précises des classes de revenus, ils confirmaient que plus le niveau économique était bas, plus l'état mental d'une population était perturbé. $\mathrm{H}$ ollingshead et $\mathrm{R}$ edlich portaient leur attention sur les populations admises en hôpital psychiatrique et ayant déjà un diagnostic. L'analyse des origines des patients leur permit de constater que les diagnostics psychiatriques variaient selon les strates de revenus. Ainsi, plus on descendait dans l'échelle des revenus, plus les diagnostics posés étaient lourds. En effet, les diagnostics de psychose, de comportement anti-sodal ou de réaction immature se concentraient parmi les patients des strates inférieures, alors que les désordres affectifs et les réactions dépressives se trouvaient parmi les patients provenant des strates supérieures (I bid:225-240). C e constat ouvrit une nouvelle piste de recherche et plusieurs études subséquentes attribuèrent les liens observés entre pauvreté et diverses psychopathologies aux inégalités des soins et services selon les classes sociales (A hr et al., 1981; Fortin, n.d.).

U ne des plus importantes études appuyant la thèse de la causalité sociale est sans doute celle menée par B renner au début des années 70 (Brenner, 1973) et elle mérite que l'on s'y attarde. Son analyse portait sur le double front des cycles économiques et des taux d'admissions psychiatriques au cours du vingtième siècle. Sauf une exception sur laquelle nous reviendrons, il constatait que chaque baisse dans l'économie était suivie de près par une hausse dans les premières admissions. De là, sa conclusion que «mental illness in A merican sodety is economic in origin» (Ibid: 8).

De ce constat, Brenner proposait certaines explications qui révèlent sa conception essentiellement fonctionnaliste du fait mental. Ainsi, pour synthétiser son argumentation, les baisses dans l'économie entraîneraient une désintégration sociale, synonyme pour lui de déviance sociale. En d'autres termes, en période de crise économique, l'individu s'avère de plus en plus incapable de remplir le rôle qui lui est attribué socialement, en particulier au niveau de son pouvoir de consommation où est 
largement déterminée sa capacité de satisfaire ses aspirations et de rencontrer ses obligations sociales. D'où les diverses manifestations de psychopathologie tels les crimes de violence, les désordres affectifs et psychosomatiques et certaines formes de turbulence politique (Ibid:6-11). C ette analyse amena Brenner à poser un jugement sévère à l'endroit de l'appareil psychiatrique qu'il considérait comme arme du système social et réponse inappropriée aux dégâts occasionnés dans la sphère économique (I bid:227-230).

$M$ ais une observation faite par Brenner lui-même vient troubler sa thèse d'une linéarité simple et directe entre économie et psychopathologie. $D$ e toutes les récessions économiques au vingtième siècle étudiées par Brenner, seule celle de la Grande D épression des années 30 ne semble pas s'être traduite en une augmentation des admissions psychiatriques (I bid:236). C eci est tout à fait surprenant car, en restant dans la logique de Brenner, on pourrait s'attendre à voir une augmentation fulgurante du nombre d'admissions. II se peut, bien sûr, que la profondeur de la crise des années 30 ait eu pour effet d'entrainer une baisse dans les budgets alloués aux développements hospitaliers, à la formation et àl'embauche d'effectifs, freinant ainsi lestaux d'admissions.

$M$ ais il se peut aussi que l'explication se trouve sur un tout autre registre. O n peut ainsi penser, par exemple, que l'ampleur du chômage et de la pauvreté dansles années 30 aurait opéré une modification au niveau des pratiques de sociabilité et des valeurs culturelles quant aux rôles sociaux et comportements prescrits.

Le travail de Brenner est d'une valeur descriptive très riche pour quiconque s'intéresse à l'histoire asilaire en Amérique du $\mathrm{N}$ ord (voir, à ce titre, et plus près de nous, I'ouvrage deA. C ellard, 1991). En revanche, la valeur explicative concernant les liensentre phénomènes mentaux et phénomènes socio-économiques et culturels enferme les thèmes récurrents de l'épidémiologie psychosociale dans une logique qui aboutit à établir une équation entre argent et bonheur - et, donc, santé mentale. C e processus de symbolisation et de représentation de la pauvreté comme problème ou risque sur le plan de la santé mentale - ou dit autrement, cette pathologisation de la pauvreté - est au coeur de la médico-psychologisation du sodal et constitue un dérapage auquel 
«C e n'est pas un hasard que ce soit dans les années 70 et 80 que la recherche sur les dimensions sociales de la problématique santé mentale se mette à la découverte de la complexité.»
Sassier, en particulier, a si bien su donner la pleine mesure (1990). Je reviendrai sur ce point en conclusion.

\section{La découverte de la complexité}

C e n'est pas un hasard que ce soit dans les années 70 et 80 que la recherche sur les dimensions sociales de la problématique santé mentale se mette à la découverte de la complexité (Elder, 1981). I ci, comme dans d'autres champs disciplinaires, l'opacité des nouvelles réalités sociales impose la remise en question d'anciens cadres conceptuels. $D$ 'où la redécouverte des théories et méthodes de l'École de C hicago des années 30 (Laperrière, 1982) et l'introduction de perspectives systémiques (Bateson, 1972) et écologiques (Bronfenbrenner, 1979) s'inscrivant dans les nouvelles thématiques du jour.

M ais si cesnouveaux courants cherchent à se démarquer de la linéarité simple d'anciensmodèles explicatifs du fait mental, leurs préoccupations profondes restent néanmoins tournées vers la compréhension des processus morbides de la psychopathologie. Dans ce sens, et à l'instar de Corin (1985), on peut dire qu'il s'agit moins d'une coupure avec le paradigme médical, que de son élargissement.

Des écrits s'inscrivant dans ces courants, on peut distinguer deux manières de concevoir la complexité. $D$ ansun cas, lesphénomènes mentaux sont étudiés comme le résultat de la conjugaison de plusieurs facteurs, internes et externes à l'individu, et qui constituent autant de maillons venant s'ajouter à la chaîne causale. Dans l'autre, il s'agit de les étudier en fonction d'un système où interagissent et s'articulent les rapports entre l'individu et les diverses composantes de son environnement.

\section{L a chaîne à maillons multiples}

Les études de ce premier type partent du constat que les variables de classe ou de sexe ne peuvent, à elles seules, expliquer l'apparente concentration des phénomènes psychopathologiques au bas de l'échelle sociale. Par exemple, si l'influente étude du M id$M$ anhattan pouvait constater que $50 \%$ de la classe inférieure 
manifestait divers degrés de troubles psychiques (Strole et al., 1962 dans G ibbs, 1980), il restait à expliquer comment l'autre $50 \%$ y échappait. D'où l'importance d'étudier les variables intermédiaires qui interviennent dans l'éclosion des problèmes mentaux parmi certains et certaines et non parmi les autres de la même catégorie sociale.

Ainsi, on met au point des échelles pour mesurer les événements de vie, positifs ou négatifs, susceptibles d'entrainer desstress et de menacer l'équilibre psychique. Et on constate que les groupes sociaux à faible revenu connaissent plus d'événements de vie négatifs tels le divorce, les discordes conjugales (D ohrenwend et D orhenwend, 1974), la perte d'emploi ou les problèmes d'argent (D ooley et $C$ atalano, 1977). M ais en raffinant les échelles de mesure, on remarque que ce sont surtout les événements négatifs ayant été vécus dans un passé lointain (Elder, 1981) ou ceux vécus de manière chronique sur une longue période qui posent le plus de danger (Belle et al., 1982). Par exemple, en étudiant une cohorte d'adultes dont l'enfance avait été marquée par la Grande D épression des années 30, Elder constate que c'est en particulier ceux qui étaient en bas âge à l'époque qui en avaient le plus souffert et dont la vie adulte montre des signes d'instabilité et d'insécurité, tant au niveau affectif que professionnel. Aussi, l'étude menée par l'équipe de Belle (1982) sur des mères de niveau socioéconomique défavorisé indique que ce sont les événements négatifs chroniques, plutôt que ceux plus récents ou ponctuels, qui font que certaines d'entre elles manifestent des signes de dépression, des problèmes d'ajustement et des difficultés dans les relations avec leurs enfants.

Un second concept qui prend de l'ampleur dans les recherches des années 70 est celui du soutien social dont on cherche à saisir le rôle dans l'équilibre psychique (D umont et al., 1988). C et intérêt ne peut être compris en dehors de l'évolution du contexte social plus large marqué, entre autres, par l'éclatement des rapports intimes, le chômage et les premières remises en question de l'Étatprovidence.

L'importance attribuée au soutien social dans l'équilibre psychique se fonde sur l'idée que face à des difficultés, le fait de 
«... face à des diffiaultés, le fait de pouvoir compter sur l'aide ou I'appui de personnes en dehors de la famille immédiate peut atténuer l'impact de la difficulté en question. » pouvoir compter sur l'aide ou l'appui de personnes en dehors de la famille immédiate peut atténuer l'impact de la difficulté en question (K aplan et al., 1977; M cK inlay, 1981 et plusieurs autres). Ainsi, en mesurant le nombre de personnes dans le réseau (parenté, amis, voisinage, etc.), certaines études ont pu établir que plus on descend l'échelle sociale, moins on semble pouvoir compter sur des ressources de soutien (Gore, 1978 dans Gibbs, 1980; $O$ uellet, 1988 dans D umont et al., 1988).

$M$ ais les mesures strictement quantitatives du soutien social posent plusieurs problèmes aux niveaux conceptuel et pratique. Par exemple, un réseau nombreux n'est pas garant d'un soutien positif, le cas du réseau du toxicomane pouvant servir ici d'exemple. Aussi, la taille d'un réseau peut diminuer selon les étapes de la vie, sans pour autant que cela puisse indiquer un problème potentiel ou réel sur le plan psychique. D 'où l'importance de considérer la densité et la qualité du réseau de soutien dansl'évaluation de sa contribution à l'équilibre mental (Barrera et al., 1981; Dumont et al., 1988). En procédant de cette manière, on peut distinguer différentstypes de soutien social: cognitif, affectif, instrumental. Ainsi, ces études tentent de montrer que dans les couches sociales dites pauvres où le réseau des individus risque fort d'être composé de personnes vivant dans les mêmes conditions, le soutien de type cognitif ou de type affectif, tels l'écoute et les conseils, sera relativement plus important que celui de type instrumental, tels la garde des enfants ou le prêt d'argent et d'autres formes d'aide concrète pouvant aider à décompresser une situation chroniquement tendue.

Sur ce point, injectons rapidement que des études récentes sur le thème de la pauvreté s'inscrivant dansune perspective théorique et méthodologique socio-anthropologique donnent un tout autre éclairage en mettant en évidence les réseaux de soutien multiples et variés qui se tissent et se reconstituent dans des conditions d'adversité souvent extrêmes. Signalons ici quelques auteures et auteurs dont les travaux constituent une contribution majeure à un corpus de connaissances grandissant qui offre un matériau empirique et théorique essentiel à la réflexion sur les pratiques d'intervention (K érisit, St-Amand et M olgat, 1994; 
Colin, O uellet, Boyer et M artin, 1991, 1992; C orin et al., 1990; Lesemann et Chaume, 1989).

$D$ 'autres études portant sur les thèmes récurrents de l'épidémiologie psychosociale mettent en évidence que dans une société de consommation, la pauvreté matérielle se traduit par une perte d'influence sociale (D ooley et C atal ano, 1977; Fried, 1985; Fortin, n.d.; et plusieurs autres). En découlent des sentiments d'infériorité et d'impuissance qui contribuent à l'émergence de symptômes d'anxiété et à la création d'un climat général de méfiance. D 'où les attitudes paranoïdes, la perte de motivation, I'affaiblissement des résistances à la frustration et la recherche de gratification immédiate. $D$ ans une recherche portant sur les familles à faible revenu d'un quartier populaire à Londres, Brown et $\mathrm{H}$ arris (1978a) ont montré comment la pauvreté contribuait à créer des relations familiales tendues. Leur recherche révèle, parmi d'autres choses, que le faible soutien du mari dans les familles ouvrières ou de milieux populaires donne lieu à une instabilité dans le couple.

Dansla même veine, Garbarino (1984) montre comment l'insécurité économique entraîne les mauvaistraitementsinfligésaux enfants de ces familles, et ce davantage dans les familles de race blanche que de race noire (Garbarino et Ebata, 1983). Cette observation n'est évidemment pas banale si on tient compte des représentations et idées reçues qui sévissent concernant ces genslà. En soi, elle constitue une piste de recherche féconde, pour peu que l'on accepte de se distancier du paradigme de la pathologie.

«C ar si les gens peuvent parfois, souvent, souffrir, entre autres, de leur condition sodale, s'ils ont parfois, souvent, besoin d'aide, ils ne sont pas nécessairement des malades... »

$C$ ar si les gens peuvent parfois, souvent, souffrir, entre autres, de leur condition sociale, s'ils ont parfois, souvent, besoin d'aide, ils ne sont pas nécessairement des malades, incapables de comprendre ce qui leur arrive, d'y donner un sens et d'y trouver des éléments de solution, toujours provisoire, imprévisible - et non planifiable.

\section{Les perspectives systémiques et écologiques}

L'émergence des perspectives systémiques et écologiques dans le champ de la santé mentale s'inscrit dans les préoccupations propres 
à nos sociétés contemporaines. Comme dans tous les champs disciplinaires, on prend conscience de l'interdépendance des phénomènes socio-culturels et de la dynamique interactionnelle et réciproque entre les composantes multiples d'un contexte pris dans son ensemble. Devant des réalités comme la pollution environnementale, la division internationale du travail, la mondialisation des économies et des marchés, les mouvements sociaux qui redéfinissent tant les rapports humains que les frontières géopolitiques, on peut dire, de manière imagée, que si un vent froid souffle dansun des quartiers du village global, tous les autres se mettent à grelotter.

C ette image vaut aussi pour les modèles systémiques et écologiques du fait mental. Ainsi, selon ces courants de pensée, les phénomènes mentaux ne peuvent être compris en dehors des relations complexes et réciproques qui se tissent dans un contexte donné. Ici, la chaîne causal e est installée dans une circularité dialectique où chaque maillon est engagé dans une réciprocité d'actions avec l'ensemble de ses composantes.

S'inspirant de la phénoménologie, G regory Bateson a été un des premiers à vouloir élaborer une théorie systémique/ écologique du fait mental (Bateson, 1972). Cherchant à comprendre la genèse de la schizophrénie, il proposait de l'analyser en fonction des codes de communication qui régissent les relations dans la famille. Pour Bateson, en somme, le schizophrène est le produit d'un système de communication truffé de messages verbaux et non verbaux contradictoires qui piègent l'individu dans une situation de double contrainte dont il ne peut sortir gagnant, quoi qu'il fasse.

La pensée de Bateson est extrêmement riche et on ne peut lui rendre justice ici. Soulignons seulement que la question des codes ou systèmes de communication est centrale à ses efforts théoriques pour comprendre les phénomènes mentaux. Pour lui, en effet, le système de codage - ou les manières de nommer les choses, le réel - s'enracine dans le système des valeurs culturelles dominantes (Bateson \& R uesch, 1988). Et l'enjeu fondamental dans la problématique de la santé mentale est celui du rapport de pouvoir qui sous- tend la légitimation d'un système de codage et 
en exclut d'autres. À ce titre, il faut souligner l'importance théorique et méthodologique de la pensée féministe quant aux rôles joués par les codes dominants dans l'exclusion des femmes et la non-reconnaissance de leur réalité (voir, par exemple, et parmi d'autres, Irigaray, 1979; O 'Brien, 1981 et 1989; Eichler, 1983; Smith et David, 1978).

$M$ ais si la pensée de Bateson ouvre sur des horizons qui permettent d'intégrer à l'analyse du fait mental les dimensions propres à divers champs de connaissance - dont l'anthropologie culturelle, la sociologie, la science politique, parmi d'autres en A mérique du $\mathrm{N}$ ord tout particulièrement, elle a donné lieu à des courants thérapeutiques beaucoup plus restreints. Ainsi, dans le cas des approches systémiques sur lesquelles se fondent les diverses thérapies familiales, le regard clinique se déplace de l'individu malade vers son contexte familial pris comme ensemble. En ce sens, on peut dire que ces courants se démarquent des approches classiques centrées sur le seul individu et sans référence à son contexte plus large. Pour ceux-là, en effet, l'individu malade est conçu comme le symptôme des relations perturbées dans sa famille. D'où la notion de l'individu-symptôme d'un milieu familial qui, lui, est malade. D'où, aussi, la nécessité de traiter l'ensemble de la famille.

Le problème qui se pose ici n'est pas celui de la frontière qui sépare l'individu de son contexte familial, mais plutôt celui d'une frontière érigée entre la famille et le contexte socio-culturel dans lequel elle s'inscrit. $\mathrm{C}$ ar, comme le fait remarquer $\mathrm{C}$ astoriadis Aulagnier, la famille peut elle-même être piégée dans un contrat social vicé d'avance et se retrouver dans la position d'exclue, d'exploitée et de victime qui lui a été imposée par la société (1981 :182192). D it autrement, et à la manière psychanalytique, si la famille peut être considérée comme une sorte de contenant qui offre un cadre de référence ou des points de repère pour contrôler les pensées potentiellement délirantes d'un de ses membres, elle est ellemême, à son tour, «contenue dans un contexte plus large qui informe ses propres réactions» (C orin, 1990:191). Faire abstraction de la réalité de l'oppression sociale sur le couple parental et du rôle qu'elle jouera dans le destin d'un des membres de la 
famille, constitue une logique qui mène inéluctablement aux diverses institutions mises en place par la société pour réparer les dommages dont elle est responsable. $D$ ans ce sens, il nous semble qu'en évacuant les dimensions socio-culturelles qui forment le contexte de la famille d'un individu-symptôme, les perspectives systémiques risquent de déboucher sur une logique institutionnelle tout à fait cohérente avec le paradigme médico-scientifique dominant.

C'est, en partie, cet ordre de préoccupations qui amenait le psychologue américain, Bronfenbrenner, à proposer un modèle écologique où les phénomènes mentaux devaient être étudiés en donnant un poids égal à l'individu et àl'environnement, particulièrement à l'interaction entre l'un et l'autre. Selon ce modèle, l'individu évolue dans un environnement tissé de relations complexes qui agissent sur lui et sur lesquelles il agit, tantôt directement, tantôt indirectement, selon le niveau des systèmes qui forment le dit environnement.

L'original ité de la la proposition de Bronfenbrenner est justement d'avoir accordé un ordre hiérarchique aux divers systèmes qui constituent l'environnement d'un individu. Ainsi, à un premier niveau, se situe le micro-système qui forme le milieu immédiat de l'individu et où se vivent ses relationsinterpersonnelles et intimes. À un second niveau, le méso-système implique I'interaction avec et entre les divers milieux que fréquente l'individu au quotidien - le travail et la famille par exemple. À un troisième niveau, l'exo-système réfère à l'interaction entre divers milieux qui n'impliquent pas l'individu de manière active, mais qui l'influencent néanmoins - par exemple, l'enfant par rapport au réseau d'amis ou au milieu de travail de ses parents. Enfin, le macro-système comprend les valeurs culturelles, les idéologies dominantes, les politiques sociales et économiques etc., qui influencent l'évolution de l'individu (B ronfenbrenner, 1979:24-27).

Selon B ronfenbrenner, une modification à l'un ou l'autre de ces niveaux aura des conséquences sur les rôles et les comportements des individus, lesquels, en retour, trouveront (ou non) leur source de légitimation dans les valeurs culturelles et l'idéologie dominante du macro-système. $\mathrm{C}$ ar c'est bien là, nous rappelle-t-il, 
«... sortir la recherche du laboratoire pour aller là où se vit le changement, car celuid constitue une véritable expérimentation prêteàporter... » que se déterminent les possibilités qu'aura l'individu de réagir aux changements dans son environnement et d'évoluer soit de manière saine, si ses rôles et comportements sont socialement acceptables, soit de manière malsaine (la soumission, la dépendance, le manque d'initiative... ), s'ils ne le sont pas (Ibid:92).

En somme, le macro-système constitue pour Bronfenbrenner la charpente de ce qu'il appelle l'environnement écologique dans lequel évolue l'individu (Ibid: 289). C ette façon de voir l'amènera à formuler un certain nombre de propositions ayant des implications sur les plans méthodologique et pratique. Ainsi, au niveau méthodologique, il se démarque à plusieurs points des approches dominantes de la recherche concernant la psychologie humaine. $R$ ésumons ce qui nous semble être les trois grandes lignes de son approche. D 'abord, il propose de sortir la recherche du laboratoire pour aller là où se vit le changement, car celui-ci constitue une véritable expérimentation prête à-porter qui comprend son propre pré- et post-test (Ibid:26-27). Ensuite, il accorde un rôle central aux perceptions subjectives des individus et à la manière dont ils définissent eux-mêmes les changements dans leur environnement (I bid:24-31 et 125). Enfin, il insiste sur l'importance de ne pas limiter la recherche à l'environnement immédiat, c'est-à-dire, au micro-système de l'individu, mais bien de l'ouvrir aux autres niveaux de son système et de les mettre en rapport les uns avec les autres (Ibid:130). En somme, et contrairement aux approches classiques pour qui l'invariance constitue la condition sine qua non du statut scientifique des hypothèses de recherche, l'approche méthodologique proposée par Bronfenbrenner permet d'intégrer les aspects non statiques qui caractérisent tout processus de changement.

M ais c'est aussi au niveau de la pratique, tant celle des intervenants professionnels que celle des planificateurs des politiques et services socio-sanitaires, que le modèle de Bronfenbrenner aura des implications importantes. Pour comprendre sa position, toutefois, il faut souligner l'influence capitale qu'ont eu sur lui les travaux de Kohn (1963), Elder (1974), O gbu (1974 dans B ronfenbrenner, 1979), qui avaient montré à quel point les comportements jugés déficitaires par les professionnels reposaient, en 
«... à défaut de pouvoir s' ouvrir aux systèmes de valeurs qui régissent les comportements, telles les relations parentsenfants des familles ouvrières ou des minorités ethniques, ce sont les valeurs inconscientes des intervenantes et intervenants qui dictent les jugements et les pratiques... » fait, sur des systèmes de valeurs propres à des groupes provenant de milieux sociaux très différents des leurs. De sorte que, à défaut de pouvoir s'ouvrir aux systèmes de valeurs qui régissent les comportements, telles les relations parents-enfants des familles ouvrières ou des minorités ethniques, ce sont les val eurs inconscientes des intervenantes et intervenants qui dictent lesjugements et les pratiques qui se veulent, par ailleurs, thérapeutiques. Ainsi, devant l'échec scolaire d'enfants pauvres et de race noire, par exemple, l'intervenant s'avérera incapable de voir qu'il s'agit peutêtre moins d'un comportement déficitaire que d'une stratégie d'adaptation de groupes marginalisés par une société qui leur offre peu d'avenir et de débouchés socio-professionnels (I bid :251).

À la lumière de ces recherches, et à l'instar de leurs auteurs, Bronfenbrenner en arrive à un rejet féroce du modèle déficitaire sous-tendant tous les niveaux de la pratique professionnelle et qu'il qualifie, sans équivoque, de socialement irresponsable (Ibid:289291). À ce modèle, il propose de substituer des approches qui font place au changement et à l'expérience du changement.

\section{En guise de conclusion: vers un déplacement du regard}

D ans les pages qui précèdent, nous avons présenté diverses thèses explicatives quant aux thèmes récurrents de l'épidémiologie psychosociale concernant notamment les milieux de pauvreté. D epuisun siècle et demi, le projet épidémiologique du fait mental a permis une accumulation imposante de connaissances qui pointent toujours dans la même direction. Avec des outils infiniment plus raffinés, on vérifie et on confirme de manière répétée et toujours plus nuancée les hypothèses scientifiques bien établies. Puisque les pauvres constituent la grande invariante de l'épidémiologie psy, c'est principalement là que se dirige le regard scientifique. Cela est cohérent avec la logique positiviste et sa manière de concevoir l'histoire des connaissances comme une 
marche inéluctable vers le progrès. A insi, on procède avec la conviction que l'on finira bien, un jour, par comprendre la nature de la bête pour pouvoir enfin la domestiquer.

Cette position épistémologique n'est ni banale, ni innocente s l'on considère le puissant dispositif technologique, thérapeutique et administratif auquel elle a donné lieu et qui en constitue, en même temps, l'impératif majeur. Sous cet angle, il nous semble que le paradigme positiviste qui forme le cadre de référence dominant dansl'étude des dimensionssociales du phénomène mental recèle des enjeux qui vont au coeur de l'éthique concernant l'égalité, la démocratie et la liberté dans nos sociétés contemporaines.

En effet, la pauvreté se présente comme un des traits saillants des phénomènes psychopathologiques au point tel que l'on peut dire qu'elle est profondément incompatible avec l'idée que l'on se fait en $O$ ccident de la santé mentale. Au rythme auquel s'étend la pauvreté dans nos sociétés, ceci a quelque chose de profondément inquiétant, à plus forte raison pour les individus et groupes coincés par les crises des temps présents et, par conséquent selon le paradigme épidémiologique, à risque sur le plan de la santé, notamment de la santé mentale, cette nomenclature moderne pour désigner le partage entre le normal et l'anormal, le sain et le pathologique.

L'inquiétude exprimée ici est bien sûre celle de voir s'étendre les malaises profonds que les conditions de contraintes extrêmes peuvent nourrir chez les individus, dans les familles et les communautés. $M$ ais sans nier cette réalité, l'inquiétude est davantage celle de voir ces malaises de base aspirés par la logique médicoadministrative de la santé publique; de voir aussi toute discussion ou publication supplémentaire mettant en évidence l'incidence et la prévalence toujours plus étendues de psychopathologies au bas de l'échelle sociale ne faire que contribuer à la stigmatisation des pauvres, à travers les notions de risque et de déterminants débouchant fatalement sur l'identification de populations-cibles ou à risque et la mise sur pied de programmes et d'interventions professionnels et techniques (R obichaud et al. , 1994:94-97). C'est dans ce sens que l'on peut dire, avec Barney (1994:31), et en écho à bien d'autres, Foucault (1972) et Illich (1976) en tête, que 
«D ans la crise actuelle des systèmes de santé et les virages importants qu'ils prennent partout, le risque est grand, comme... de se tourner vers une gestion de la pénurie plutôt que vers une redéfinition de notre philosophie de la santé. » le système de santé mentale «draws additional strength from the mere existence of the growing misery accompanying the deterioration of W estern society and late capitalism». D ans la crise actuelle des systèmes de santé et les virages importants qu'ils prennent partout, le risque est grand, comme l'a si justement soulignéW illie Appollon, de se tourner vers une gestion de la pénurie plutôt que vers une redéfinition de notre philosophie de la santé (A pollon, 1995).

D epuis une quinzaine d'anées en particulier, on assiste à des transformations profondesà tous les niveaux du tissu social. Q u'il s'agisse des psychiatrisés désinstitutionnal isés, des immigrants de race, culture et langue étrangères fuyant des pays déchirés par la guerre et le sous- développement, des familles brisées, des sansemploi, des gais, des gangs, des sans-abri, des personnes âgées, des pauvres, anciens et nouveaux... bref, ces catégories sociales récemment apparues font éclater l'illusion d'une société intégrée, monolithique, harmonieuse et témoignent de l'émergence de nouvelles couches d'exclu-e-s et de marginalisé-e-s. Si chaque époque et chaque société se donnent des normes pour marquer le départage entre cas et non-cas, les nôtres, nous rappelle Foucault (voir D reyfus et $\mathrm{R}$ abinow, 1984), sont devenues exeptionnellement dangereuses parce que de plus en plus globalisantes et homogénéisantes. II est important de rappeler que l'épidémiologie psychosociale (dont les grilles, classifications et instruments de mesures sont d'origine américaine, quand ce n'est copie conforme) constitue un outil central dansla définition des besoins, la planification des services et la formulation des politiques de santél santé mentale partout en Amérique du $\mathrm{N}$ ord, au Canada, en O ntario et ailleurs. C'est, du reste, son intention explicite que I'on reconnaît généralement sous le vocable approximatif d'aide aux prises (ou non-prises) de déision. $D$ ans ce processus, les études qui mettent en évidence les phénomènes pathologiques dans les marges sociales constituent des systèmes de marquage qui s'avèrent d'une importance capitale pour légitimer tout un dispositif de services et d'interventions. Dans ce sens, les construits nosologiques déterminés d'avance ne s'avèrent pas seulement incapables de traduire ces réalités autres; plus fondamentalement, ils contribuent à les taire et, ce faisant, à dissimuler une intolérance infiniment plus subtile. 0 n peut se demander, à l'instar de Sassier, 
«... comprendre le rôle des facteurs socioalturels, non pas tant dans l'émergence des phénomènes mentaux, mais davantage dans la manière dont ils sont représentés, véaus et exprimés dans des contextes spécifiques et différents » si cette symbolisation de la pauvreté comme risque ou problème sur le plan de la santé mentale, avec tout le dispositif institutionnel qui s'ensuit, ne constitue pasla forme moderne de l'enfermement du fait mental.

D ans ce contexte, les courants de recherche inspirés surtout, mais non pas uniquement, par l'anthropologie, la sociologie, le féminisme, ainsi que par certaines notions proposées par Bateson et Bronfenbrenner concernant les valeurs qui sous-tendent les systèmes de codage utilisés pour définir les problèmes et besoins, nous paraissent particulièrement féconds pour une démarche qui cherche à comprendre le rôle des facteurs socio-culturels, non pas tant dans l'émergence des phénomènes mentaux, mais davantage dans la manière dont ils sont représentés, vécus et exprimés dans des contextes spécifiques et différents. U ne démarche qui cherche également à voir non pas tant la victime, que l'acteur capable de comprendre ce qui lui arrive, d'y donner un sens et d'aménager des contraintes parfois extrêmes. $C$ et effort pour se décentrer par rapport aux idées reçues du savoir professionnel et se placer du point de vue des gens qui subissent de plein fouet les crises actuelles permettrait de penser, à la suite de B ourdieu, que si nous nous retrouvions dans les mêmes situations, nous ferions peut- être pareil (Bourdieu, 1991:5). Et en bout de ligne, ce regard autre ouvrirait l'horizon sur des pratiques moins technicoprofessionnelles, et plus de l'ordre de la citoyenneté et de la solidarité. $\mathrm{O}$ u, dit autrement, des pratiques vues comme aide à des personnes en danger, et en fonction de critères d'urgence (Ewald, 1993).

\section{Bibliographie}

AHR, P.R ., M.J. GORODEZKY et D.W. CHO (1981). «M easuring the R elationship of Public Psychiatric Admissions to $\mathrm{R}$ ising U nemployment», $\mathrm{H}$ ospital and $\mathrm{C}$ ommunity Psychiatry, vol. 32, no 6, 397-401.

AMER ICAN PSY CHIATR IC ASSO CIATION (1994). D iagnostic and Statistical M anual of M ental D isorders (D SM -IV), Washington, A merican Psychiatric Association.

APO LLO N . W. (1995). «Du spécifique à l'universel: entre sens et contresens», C ommunication au colloque $L$ a dinique au pluriel: ancrages métissages et ruptures $C$ olloque du $G$ roupe interuniversitaire de recherche en anthropologie médicale et ethnopsychiatrie (GIR AM E), M ontréal, le 21 et 22 avril. 
BAR N EY, K. (1994). « Limitations of the Critique of the M edical M odel», The Journal of M ind and $B$ ehavior. $C$ hallenging theT herapeutic State, part two: $F$ urther $D$ isquisitions on the $M$ ental $H$ ealth System, vol. 15, no 1-2, 19-34.

BAR R ER A, M . (1986). «Distinctions Between Social Support Concepts, M easures and M odels», A merican Journal of $C$ ommunity Psychology, vol. 14, no 4, 413-445.

BAR R ER A, M., I.N. SAN D LER et T.B. R AM SAY (1981). «Preliminary D evelopment of a Scale of Social Support: Studies on College Students», A merican Journal of C ommunity Psychology, vol. 9, no 4, 435-447.

BAST IDE, R . (1965). Sociologie des maladies mentales, Paris, Flammarion.

BAT ESO N , B. (1972). StepsTowards an E cology of M ind, N ew York, Ballantine Books.

BATESO N, G. et J.R . RU ESCH (1988). Communication et Sodété, Paris, Seuil.

BEBBINGT O N, P., J. HURRY, C.TEN N AN T, E. ST UR T et J.K. KIN G (1981). «Epidemiology of $M$ ental Disorders in C amberwell», Psychological M ediane, vol. 11, 561-579.

BELLE, D., C. LON GFELLOW,V. PAR KER MAKOVSKY (1982). «Stress, Depression and the $M$ other-C hild R elationship: R eport of a Field Study», International Journal of Sodology and the Family, vol. 12, 251-263.

BER BER O VA, N . (1988). Le roseau révolté. Arles, A ctes Sud.

BIBEAU, G., C. SABATIER, E. COR IN , M. TOUSSIGN AN T et J.F. SAU CIER (1989). «a recherche anglo-saxonne en santé mentale: tendances, limites et impasses». Santé M entale au $Q$ uébec, vol. 14, no 1, 103-121.

BLAIS, L. (1987). «À propos de la santé mentale et des processus sociaux. N ote critique», Santé mentale au Q uébec, vol. 12, no 1, 175-177.

BLAIS, L., (1993). L'étrangère à nos portes Femmes, pauvretés et santé mentale, Thèse de doctorat, U niversité de M ontréal.

BO U R DIEU, P. (1991). «ntroduction à la socioanalyse», A des de la recherche en siences sodiales, no 90, 3-19.

BR EN N ER , M .H . (1973). M ental IIIness and the E conomy, C ambridge, H arvard U niversity Press.

BRONFEN BR EN NER, U. (1979). T he ecology of human development: Experiments by nature and design, $\mathrm{C}$ ambridge, $\mathrm{H}$ arvard $U$ niversity Press.

BR OW N , G.W. etT. H AR R IS (1978a). Sodal O rigins of D epression: A Study of P sychiatric D isorder in Women, London, Tavistock.

BR OW N, G.W. et T. HAR R IS (1978b). «Social O rigins of Depression: A R eply», Psychological M ediane, vol. 8, 577-588.

CAST EL, F., R . CAST EL et A. LOVELL (1979). La société psychiatrique avancée, le modèle américain, Paris, Grasset.

CAST O R IADIS-AU LAGN IER , P. (1981). La violence de l'interprétation: du pidogramme à l'énoncé, Paris, P.U.F., coll. le fil rouge.

CELLAR D,A. (1991). H istoire de la folie au Q uébec de 1600 à 1850, M ontréal, Boréal.

COLIN, C., F. OUELLET, G. BOYER et C. MARTIN (1991). Survivre et penser à sa santé en situation d'extrême pauvreté: le point de vue des femmes enceintes de M ontréal, DSC M aisonneuveR osemont et D SC Sacré C oeur, M ontréal.

COLIN , C., F. O U ELLET, G. BOYER et C. MARTIN (1992). Extrême pauvreté, maternité et santé, M ontréal, Éditions St-M artin. 
CO R IN , E. (1985). »La santé: nouvelles conceptions, nouvelles images», dans ). D ufresne, F. D umont et Y. M artin, éd.T raité d' anthropologie médicale, Q uébec, Presses de I'U niversité du Q uébec, 45- 73.

CORIN, E., G. BIBEAU, J.C. M ARTIN et R . LAPLAN TE (1990). Comprendre pour soigner autre ment, M ontréal, Les Presses de L'U niversité de M ontréal.

CO R IN , E. (1990). « es dynamiques familiales et leur étayage culturel dans le cas de la schizophrénie», dans C. Brodeur, R. Pelsser et G.Tarrab, éd. La Famille: I'individu-plus-un, approche psychanalytique et approche systémique, O ttawa, Éditions G.Vermette Inc., 161-203.

DE GAU LÉJAC, V. (1987). La névrose de dasse, Paris, H ommes et Groupes éditeurs.

DOHR EN W EN D, B.S. et B.P. DOHR EN WEN D (eds) (1974). Stresfful Life E vents: T heir N ature and $\mathrm{E}$ ffects, $\mathrm{N}$ ew York, J. W iley and Sons.

DO O LEY, R . et C.D.C ATA LAN O (1977). «Economic predictions of depressed mood and stresfful life events in a metropolitan community», Journal of $H$ ealth \& Sodal Behavior, vol. 18, 292-307.

DR EY FUS, H. et P. R ABIN OW (1984). M ichel F oucault, un parcours philosophique, Paris, $G$ allimard.

DU M ON T, M ., L. BLAN CHET et P.H.TR EM BLAY (1988). I solement et santé mentale (suffit-il de quitter son île?): R evue de la littérature, M ontréal, H ôpital du Sacré-C oeur, D épartement de santé communautaire, module Santé mentale, M ontréal.

EIC H LER , M . (1983). Families in C anadaToday: $R$ ecent $C$ hanges andT heir Policy $C$ onsequences,Toronto, Gage Publishing.

ELDER , G.H . (1981). «H istory and the Family:The D iscovery of Complexity», Journal of M arriage and the Family, Août, 489-519.

EWALD, F. (1993). «L'État providence», Les temps modernes, vol. 567, 252-255.

FAR IS, R .E.L. and H.W. DUN HAM (1939). M ental disorders in U rban A reas, Chicago, U niversity of Chicago Press.

FO RTIN, D. (non daté). L a pauvreté et la maladie mentale: Est-œe que les pauvres sont plus malades et si oui pourquoi?, U Q AM , D épartement de psychologie, texte ronéo-typé.

FO R TIN , D. (1987). «Variables économiques et santé mentale», dans J. Guay, ed. M anuel québécois de psychologie communautaire, Chicoutimi, Gaëtan M orin éditeur, 133-157.

FO U C AU LT, M . (1972). H istoire de la folie à I'âge classique, Paris, Gallimard.

FR IED, M . (1985). «Social Differences in M ental H ealth», dans J. Kosa et I.K . Z ola éds. Poverty and $H$ ealth, édition révisé, Boston, $\mathrm{H}$ arvard U niversity Press, 135- 192.

GAR BAR IN 0, J. (1984). «C hildW elfare and the Economic C rises», C hild W elfare, vol. 63, no 1, 3- 15.

GAR BAR IN O, J. et A. EBATA (1983). «T he significance of ethnic and cultural differences in child maltreatment», Journal of $M$ arriage \& the Family, novembre, 773-783.

GIBBS, M .S. (1980). «Social class, mental disorder and the implications for community psychology», dans M .S. Gibbs, J.R . Lachenmeyer et J. Segal, éds., C ommunity P sychology:T heoretical and E mpirical A pproaches, N ew York, Gardner Press Inc., 173-205.

GU ELFI, J.D. (1984). «D’Esquirol au DSM III: les avatars de la nosographie», P rospedive et Santé, no 29, 89-95.

HOLLIN GSHEAD,A.B. and F.C. REDLICH (1958). Sodal class and mental illness: A Study, N ew York, Wiley.

H U SAIN I, B.A. et J.A. N EFF (1981). «Social C lass and D epressive Symptomatology, The R ole of Life Change Events and Locus of C ontrol», Journal of N ervous and M ental D isorders, vol. 169, no 10, 638-647. 
ILLIC H, I. (1976). N émésis médical, l'expropriation de la santé, Paris, Seuil.

IR IGAR AY, L. (1979). Et I' une ne bouge pas sans I'autre, Paris, Éditions de minuit.

KAPLAN, B.H., J. CASSEL et S. GR OVE (1977). «Social Support and H ealth», M edical C are, vol. 15 (Supplément au no 5), 47-58.

KÉR ISIT, M., N. ST-AM AN D et M . M O LGAT (1994). "Id, c'est chez nous" R essources alternatives pour familles démunies, $\mathrm{O}$ ttawa, École de service social, U niversité d'O ttawa.

LALO N DE, M. (1974). N ouvelles perspectives de la santé des C anadiens, $O$ ttawa, Santé et Bien-être C anada, avril.

LAPER R IĖR E, A. (1982). «Pour une construction empirique de la théorie: la nouvelle École de Chicago», Sociologie et Sodétés, vol. 14, no 1, 31-42.

LEIGHTON, A.H. (1959). M y name is legion: Foundations of a theory ou man in relation to culture, Volume 1,T he Stirling C ounty study of psychiatric disorder and sociocultural environment, $\mathrm{N}$ ew York, Basic Books.

LESEM AN, F. et C. CH AU M E (1989). F amilles-providence, la part de l'É tat. R echerches sur le maintien à domiale, M ontréal, É ditions St-M artin.

LIEM , R . and J. LIEM (1978). «Social class and mental health reconsidered:The role of economic stress and social support», Journal of H ealth and Social B ehavior, vol. 19, 139-156.

M C KIN LAY, J.B. (1981). «Social N etwork Influences on M orbid Episodes and the C areer of $\mathrm{H}$ elp Seeking», dans L. Eisenberg et A. K lerman, éd.T he R elevance of Social Science for M ediane, B oston, R eidel Publishing C0., 77-107.

O 'BR IEN , M . (1981). The Politics of R eproduction, London, R outledge and Kegan Paul.

$O$ 'BR IEN , M . (1989). R eproduang the world. E ssays in feminist theory, London, Westview Press.

PEAR LIN, L.I. et L. SC HOO LER (1978). «T he Structure of C oping», Journal of H ealth \& Sodal Behavior, vol. 19, 2-21.

ROBICHAU D, J.-B., L. GUAY, C. COLIN, M. POTHIER et J.-F. SAU CIER (1994). Les liens entre la pauvreté et la santé mentale $D$ e l'exdusion à l'équité, Le comité de la santé mentale du $Q$ uébec, $M$ ontréal, Gaëtan $M$ orin éditeur.

ROBIN S, L.N ., B.Z. LO CKE et D.A.R EGIER (1991). «An overview of psychiatric disorders in A merica». dans L.N. R obins et D.A. R egier, eds. P sychiatric disorders in A merica. T he E pidemiologic $C$ atchment A rea Study, N ew York, The Free Press, 328-366.

SAR BIN , T.R . (1990). «Toward the O bsolescence of the Schizophrenia H ypothesis». Journal of $M$ ind and B ehavior. $C$ hallenging theT herapeutic $S$ tate: $C$ ritical Perspectives on P sychiatry and the $M$ ental H ealth System. (numéro spécial dirigé par D. C ohen). vol. 11, no 3 et 4, 259-283.

SASSIER , P. (1990). D u bon usage des pauvres: $H$ istoire d'un thème politique, XV I - X X e siècle, Paris, Fayard.

SM IT H, D.E. et S.J. DAVID (1975). W omen L ook at Psychiatry, Vancouver, Press G ang Publishers.

STATISTIQU E CANADA (1981). La Santé des C anadiens, R apport de l'E nquête Santé C anada, $O$ ttawa, Santé et bien-être social $C$ anada.

T O U SIGN AN T, M. (1987). L'étiologie sociale en santé mentale, U niversité du Q uébec à M ontréal, Larehs, document déposé au C.Q.R.S., juin. 